\title{
Ramos colaterais do arco aórtico e suas principais ramificações em nutria (Myocastor coypus)
}

\author{
Collaterals branches of the aortic arch and its main rami in nutria (Myocastor coypus) \\ Rui Campos ${ }^{1,2}$, Ana Cristina Pacheco de Araújo ${ }^{3}$ \& Rodrigo Cavalcanti de Azambuja ${ }^{3}$
}

\begin{abstract}
Background: Nutria (Myocastor coypus), also known as Swamp Beaver, is a medium-sized semiaquatic rodent that belongs to the Capromyidae family. Originally from the southernmost part of South America, the species is distributed in several parts worldwide such as Europe and United States, where it has been used for commercial purposes due to the excellent quality of its fur and meat. Information about the nutria morphology is rare. Only a few articles about its abdominal aorta branches can be found, but nothing exists regarding its aortic arch. Consequently, other rodents such as chinchillas, agoutis, guinea pigs capybaras, pacas and rats will be used in the discussion. Therefore, this study aims to obtain morphological information that could justify such discussions in a functional point of view, and that could result in support for a better understanding of the physiology of this animal.

Materials, Methods \& Results: Thirty-two Myocastor coypus were used in the study, originated from a breeding facility in the town of Caxias do Sul, RS and authorized by IBAMA. The animals were put to sleep by means of an anesthetic overdose administrated intraperitoneally, and kept in formaldehyde for seven days to be subsequently dissected. After having their arterial system flushed with saline solution, the aorta of thirty specimens received an injection containing latex 603 through the left ventricle, for later observation of the arteries of the cranial mediastinal space and neck. Dental resin was injected in two specimens, for subsequent manufacture of molds by means of maceration. Schematic drawings of all parts were made with the help of a magnifying glass, for posterior composition of results. The brachiocephalic trunk and the subclavian artery arose in sequence from the aortic arch of the nutria in $60 \%$ of the samples, whereas the brachiocephalic trunk, left common carotid artery and left subclavian artery arose from the arch in $40 \%$ of the samples. The branching sequence of the collateral branches of the subclavian arteries showed a great variation, presenting isolated vessels and forming trunks among the arteries identified (according to the tables). The thoracic vertebral, vertebral, internal thoracic, dorsal scapular arteries and the superficial-deep cervical trunk aroused medially from the right subclavian artery towards a lateral direction, as main collateral branches of highest prevalence. On the other hand, the left subclavian artery also gave off the vertebral artery as its first vessel, followed by the internal thoracic and thoracic vertebral arteries, and its last collateral branch was a common trunk between the dorsal scapular artery and the superficial-deep cervical trunk.

Discussion: Other rodents in the study presented the same aortic arch sequence as observed in nutrias i.e., the brachiocephalic trunk and the left subclavian artery. But the branches of these main vessels showed remarkable differences, with the formation of several common trunks among the arteries. Additionally, the origin sequence of these vessels was different in the rodents studied. Therefore, the aortic arch of nutrias has two model patterns for its branching. In $60 \%$ of the samples, the aortic arch gives off the brachiocephalic trunk and the left subclavian artery as collateral branches. In $40 \%$ of the samples, however, the branching sequence is composed of the brachiocephalic trunk, the common left carotid artery and the left subclavian artery.
\end{abstract}

Keywords: aortic arch, artery vascularization, rodentia.

Descritores: arco aórtico, vascularização arterial, roedores. 


\section{INTRODUÇÃO}

A nutria (Myocastor coypus), também conhecida como ratão-do-banhado, é um roedor semi-aquático de tamanho mediano e que pertence à família Capromyidae. Ao atingir a fase adulta, poderá pesar entre seis e nove quilos e ter $60 \mathrm{~cm}$ de comprimento. Originária do extremo sul da América do Sul, está disseminada em várias partes do mundo como Europa e EUA, onde tem sido explorada com fins comerciais devido à excelente qualidade de pelagem e carne [2]. São animais que se alimentam de capim, raízes e plantas aquáticas e têm a pele recoberta por longos pelos de coloração marrom escuro e amarelo claro. Vivem em banhados, rios e lagos, onde cavam tocas ao longo das margens que servem de refugio ou ninho [11].

Informações sobre a morfologia da nutria são raras, sendo encontrados apenas alguns artigos sobre a ramificação da artéria aorta abdominal, mas nada em relação ao arco aórtico. Devido a isso, outros roedores serão utilizados na discussão, como: chinchila [1], cutia [3], porquinho-da-índia [6,9,10], capivara [7, 4], paca [8] e rato [12]. Desse modo, este trabalho busca obter informações de cunho morfológico que possam fundamentar discussões do ponto de vista funcional e que resultem em subsídios para a melhor compreensão da fisiologia destes animais. Assim, é possível oferecer suporte para medidas que visem a protegê-los em seu ambiente natural, bem como aprimorar métodos de sua exploração racional. Portanto, o presente trabalho tem como objetivo sistematizar e descrever os ramos colaterais do arco aórtico e suas principais ramificações em nutria.

\section{MATERIAIS E MÉTODOS}

Foram utilizados 32 exemplares de Myocastor coypus, provenientes de criatório do município de Caxias do Sul, RS, autorizado pelo IBAMA, sendo 15 machos e 15 fêmeas para a sistematização e dois exemplares utilizados para confecção de molde em acrílico.

Para o preparo das peças, os animais receberam 10000 UI de heparina ${ }^{1}$ e 30 minutos depois, 20 $\mathrm{ml}$ de Thiopental sódico ${ }^{2}$ a $12,5 \%$, ambos pela via intraperitoneal. A cavidade torácica foi aberta ventralmente em plastrão, a artéria aorta torácica clampeada próximo ao diafragma, o saco pericárdico aberto, o ápice cardíaco seccionado e a artéria aorta canulada via ventrículo esquerdo.

O sistema arterial de 30 espécimes foi lavado com $150 \mathrm{ml}$ de solução salina resfriada a $0,9 \%$, seguido de preenchimento com látex $603^{3}$ corado em vermelho. $\mathrm{O}$ animal permaneceu em água corrente por uma hora para polimeralização do látex, a pele foi rebatida e as peças foram fixadas em formaldeído a $20 \%$ por sete dias, para posterior dissecção e observação das artérias do espaço mediastínico cranial e pescoço. Em dois animais injetou-se resina odontológica ${ }^{4}$ corada com pigmento específico para confecção de moldes vasculares, por maceração durante seis meses.

Desenhos esquemáticos de todas as peças foram elaborados com auxílio de uma lupa ${ }^{5}$ para posterior confecção dos resultados. Alguns exemplares foram fotografados ${ }^{6}$ para documentação e os vasos nominados de acordo com a NOMINA ANATÔMICA VETERINÁRIOS (2005). A análise estatística dos resultados constou da aplicação do cálculo de percentagem.

\section{RESULTADOS}

O arco aórtico da nutria apresentou-se com uma curvatura à esquerda, em todas as peças, e emitiu como seus ramos colaterais, em sequência, o tronco braquiocefálico e a artéria subclávia esquerda em 60\% das amostras (Figura 1A). Já em 40\% das preparações, a sequência de ramos encontrados foi um tronco braquiocefálico, artéria carótida comum esquerda e artéria subclávia esquerda (Figura 1B). Assim, a nutria apresentou dois padrões de ramificação do arco aórtico.

O tronco braquiocefálico emitiu, em $60 \%$ das amostras, a artéria carótida comum esquerda, a alguns milímetros depois, a artéria carótida comum direita, continuando como artéria subclávia direita (Figura 1A). Já em 40\% dos achados, o tronco emitiu, a alguns milímetros de sua origem, a artéria carótida comum direita, continuando como artéria subclávia direita (Figura 1B).

As artérias carótidas comuns, direita e esquerda, ascenderam o pescoço acompanhando a traquéia, lateralmente, alcançando a base do crânio, dividindo-se na altura da alça do nervo hipoglosso (XII par craniano) em uma artéria occipital, continuando para 

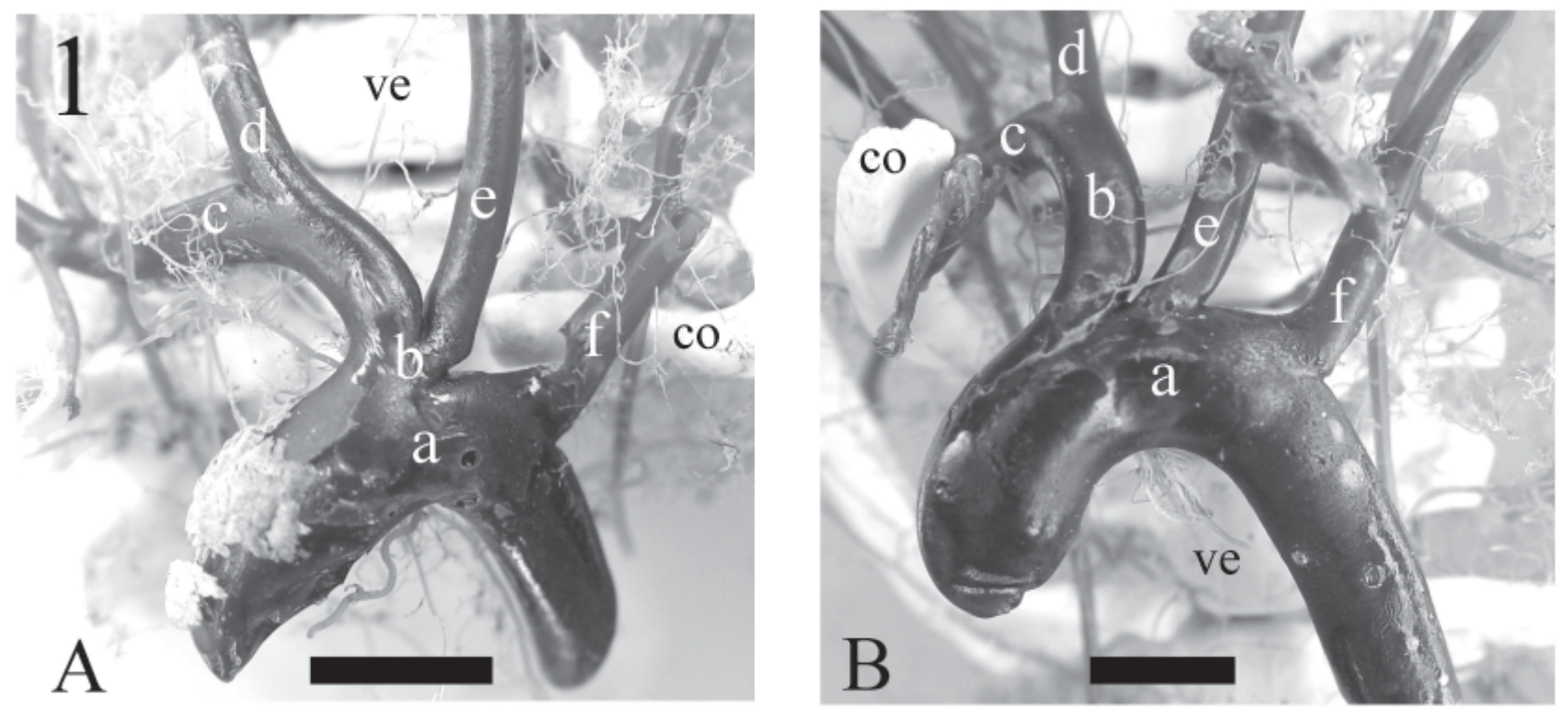

Figura 1. Fotografia em vista ventral dos modelos em acrílico, representando, em A e B, os dois modelos padrões de ramificação do arco aórtico em nutria: a - arco aórtico; b - tronco braquiocefálico; c - artéria subclávia direita; d - artéria carótida comum direita; e - artéria carótida comum esquerda; f - artéria subclávia esquerda; co - costela; ve - vértebra cervical. Barra em A e B $=7 \mathrm{~mm}$.

a face como artéria carótida externa. A artéria occipital emitiu uma fina artéria carótida interna que se dirigiu ao forame lácero, medialmente a bolha timpânica, capilarizando-se em $100 \%$ das peças, não participando da irrigação encefálica.

A artéria subclávia direita emitiu, de medial para lateral, como ramos colaterais principais às artérias: vertebral torácica, vertebral, torácica interna, escapular dorsal, tronco comum cervical superficialprofunda (Figura 2A). Já a artéria subclávia esquerda emitiu, também de medial para lateral, como ramos colaterais principais as artérias: vertebral torácica, torácica interna, vertebral, tronco escapular dorsal com tronco comum cervical superficial-profunda (Figura 2B). Tanto à direita como à esquerda, esses ramos colaterais foram emitidos em uma sequência de vasos isolados ou formando troncos compostos de formas variadas.

A artéria vertebral torácica foi lançada da artéria subclávia, caudalmente, de maneira individual ou em tronco comum, sendo dela emitidas as três primeiras artérias intercostais dorsais. À direita, a artéria vertebral torácica apresentou-se como ramo individual da artéria subclávia em 53,4\% das amostras, sendo primeiro ramo em $36,7 \%$ e segundo ramo em $16,7 \%$ das peças. Já em $46,6 \%$ dos casos, a artéria vertebral torácica foi lançada da artéria subclávia direita em tronco comum com diversas artérias, conforme descrito na Tabela 1. À esquerda, a artéria subclávia emitiu a artéria vertebral torácica de maneira pouco variada, sendo sempre primeiro ramo colateral. Individualmente, foi observada em 93,3\% das amostras e em tronco comum em 6,7\% das preparações, conforme Tabela 1.

A artéria vertebral foi projetada crâniodorsalmente da artéria subclávia, incorporou-se ao forame transverso da sexta vértebra cervical, ascendeu o canal transversal e, ao ultrapassar o forame transverso do Atlas, atingiu a fossa atlantal, penetrando rostralmente no forame alar e vertebral lateral para o interior do canal vertebral, indo anastomosar-se com sua homóloga contralateral, na face ventral da medula oblonga, formando a artéria basilar. Por sua vez, a artéria basilar foi responsável pela irrigação encefálica em todos os casos. À direita, a artéria vertebral foi emitida como ramo colateral individual em $70 \%$ das amostras, sendo primeiro ramo em $26,7 \%$, segundo ramo em $36,6 \%$ e terceiro ramo em $6,7 \%$ das amostras. Já em $30 \%$ dos casos, a artéria vertebral foi lançada pela artéria subclávia direita em tronco comum com outros vasos, sendo primeiro ou segundo ramo colateral conforme Tabela 2. À esquerda, a artéria vertebral foi emitida como ramo individual em $63,4 \%$, sendo segundo ramo em $16,7 \%$ e terceiro ramo em $46,7 \%$ das amostras. Ainda em 36,6\% das preparações, a artéria vertebral foi lançada pela artéria sub- 

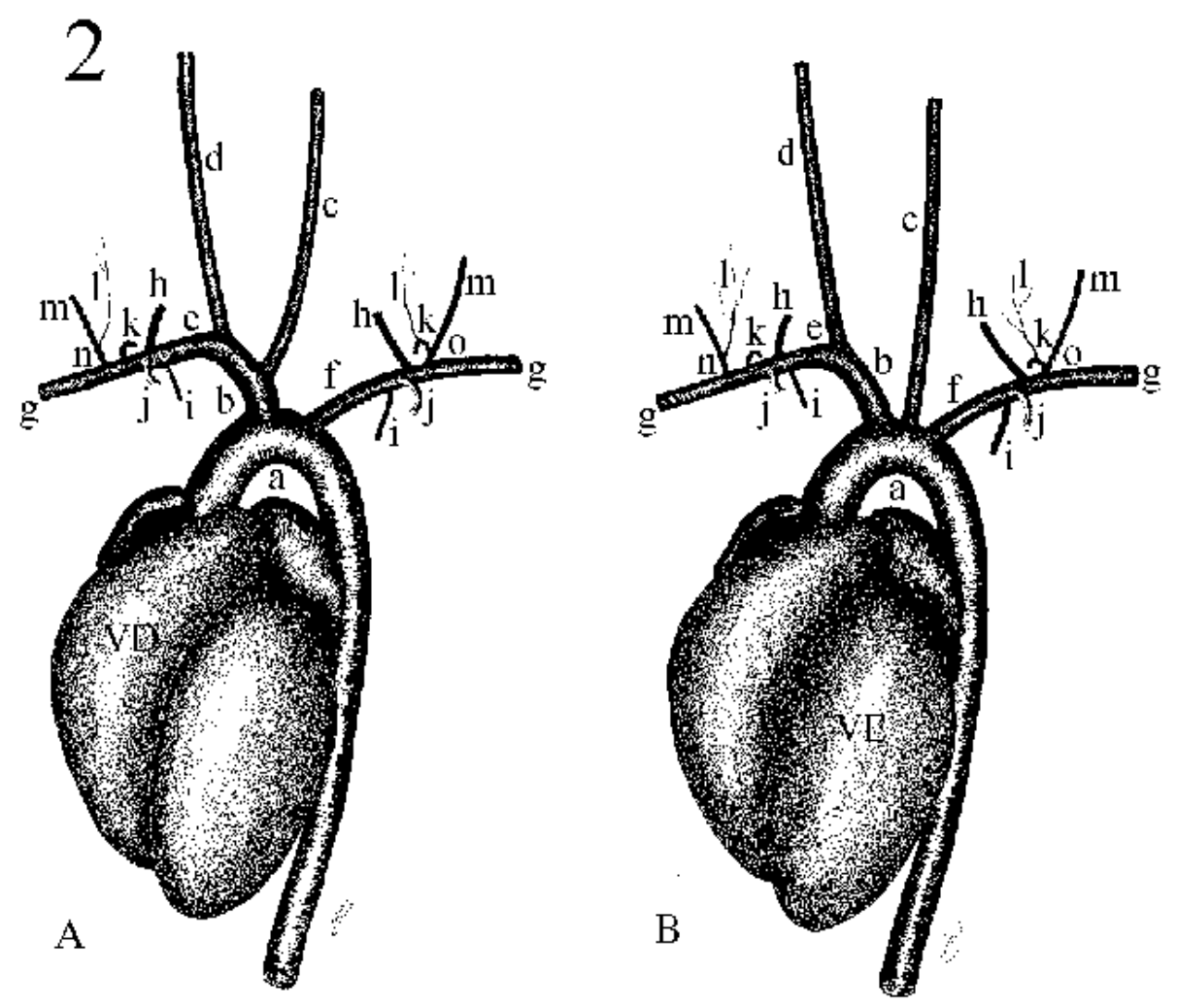

Figura 2. Desenhos esquemáticos em vista ventral do coração e do arco aórtico da nutria e suas principais ramificações: A - esquema padrão de $60 \%$; B - esquema padrão de 40\%; a - arco aórtico; b - tronco braquiocefálico; c - artéria carótida comum esquerda; d - artéria carótida comum direita; e - artéria subclávia direita; f - artéria subclávia esquerda; g - artéria axilar; $\mathrm{h}$ - artéria vertebral; i - artéria vertebral-torácica; $\mathrm{j}$ - artéria torácica interna; $\mathrm{k}$ - artéria escapular dorsal; 1 - artéria cervical-profunda; $\mathrm{m}$ - artéria cervical superficial; $\mathrm{n}$ - tronco comum cervical superficial-profunda; o - tronco escapular dorsal com tronco comum cervical superficial-profunda; VD - ventrículo direito; VE - ventrículo esquerdo.

clávia esquerda em tronco comum com outros vasos, variando sua sequência de origem de primeiro até quarto ramo, conforme Tabela 2.

A artéria torácica interna foi lançada ventralmente pela artéria subclávia percorrendo, caudalmente, entre as cartilagens costais e o músculo transverso torácico, emitindo todos os ramos intercostais ventrais, indo atingir a parede abdominal como artéria epigástrica superficial cranial. À direita, foi emitida individualmente em $73,4 \%$ das peças, variando sua ordem de emissão de segundo a quinto ramo, conforme Tabela 3. Já em tronco comum, foi observada em $26,6 \%$ das peças, sendo primeiro ramo colateral da artéria subclávia direita, em tronco comum com as artérias vertebral torácica e escapular dorsal, em 3,3\% e, somente com a artéria vertebral torácica, em $13,3 \%$ das amostras. Ainda à direita, foi emitida como segundo ramo em tronco comum com as mesmas artérias supracitadas, sendo em $6,7 \%$ com a artéria vertebral torácica e em 3,3\% das amostras com as artérias vertebral torácica e escapular dorsal. À es- querda, a artéria torácica interna foi emitida da artéria subclávia somente de maneira individual, variando também sua ordem de emissão conforme Tabela 3 .

A artéria escapular dorsal projetou-se dorsalmente, indo irrigar a musculatura profunda da goteira vertebral, na região escapular. Apresentou uma variação considerável na sequência de emissão como ramo colateral individual e como tronco comum com outras artérias, tanto à direita como à esquerda. A artéria escapular dorsal foi lançada pela artéria subclávia de maneira individual à direita em 36,7\% das amostras enquanto à esquerda em $33,4 \%$, conforme a Tabela 4. Essa mesma artéria também se apresentou formando troncos comuns com outras artérias, sendo à direita em 63,3\% e à esquerda em 66,6\%, de acordo com a Tabela 5.

A artéria cervical profunda, de pequeno calibre, foi emitida dorsocranialmente irrigando a musculatura profunda ventral do pescoço. A artéria subclávia, em ambos os antímeros, não emitiu, indi- 
Tabela 1. Frequência de emissão da artéria vertebral torácica em tronco comum com outras artérias, como ramo colateral da artéria subclávia, à direita e à esquerda.

\begin{tabular}{cccc}
\hline Tronco comum com: & \multicolumn{1}{c}{ como $\mathbf{1}^{\mathbf{0}}$ ramo } & como $\mathbf{2}^{\mathbf{0}}$ ramo \\
\hline & A. S. D. & A. S. E. & A. S. D. \\
Aa T.I. \& E.D. & $3,30 \%$ & $3,30 \%$ \\
A. T.I. & $13,30 \%$ & $6,70 \%$ \\
A. Ve. & $6,70 \%$ & $3,30 \%$ & \\
Aa. Ve., E.D. \& Tc.C.S-P & $3,30 \%$ & & $10 \%$ \\
A. E.D. & & $3,40 \%$ & \\
\hline
\end{tabular}

Tabela 2. Frequência de emissão da artéria vertebral em tronco comum com outras artérias, como ramo colateral da artéria subclávia, à direita e à esquerda.

\begin{tabular}{|c|c|c|c|c|c|}
\hline Tronco comum com: & como $1^{\circ}$ ramo & como 2 & ${ }^{\circ}$ ramo & $\operatorname{como} 3^{\circ}$ ramo & como $4^{\circ}$ ramo \\
\hline & A. S. D. A. S. E. & A. S. D. & A. S. E. & A. S. E. & A. S. E. \\
\hline A. V. T. & $6,70 \% \quad 3,30 \%$ & & & & \\
\hline Tc. C.S-P & $6,70 \%$ & & & $3,30 \%$ & $3,30 \%$ \\
\hline A.E.D. \& Tc.C.S-P & $3,30 \%$ & $6,70 \%$ & & $6,70 \%$ & \\
\hline $\begin{array}{l}\text { Aa V.T., E.D. \& } \\
\text { Tc.C.S-P }\end{array}$ & $3,30 \%$ & & & & \\
\hline Aa E.D. \& C.P. & & $3,30 \%$ & $3,30 \%$ & & \\
\hline A. E. D. & & & $6,70 \%$ & $3,30 \%$ & \\
\hline A. C. P. & & & & $3,30 \%$ & $3,30 \%$ \\
\hline
\end{tabular}

Tabela 3. Ordem de emissão da artéria torácica interna como ramo colateral individual da artéria subclávia, à direita e à esquerda.

\begin{tabular}{ccc}
\hline Ordem de emissão & \multicolumn{2}{c}{ Individualmente } \\
\hline \multirow{2}{*}{$2^{\circ}$ ramo } & direita & esquerda \\
$3^{\circ}$ ramo & $6,70 \%$ & $66,70 \%$ \\
$4^{\circ}$ ramo & $26,70 \%$ & $23,30 \%$ \\
$5^{\circ}$ ramo & $26,70 \%$ & $6,70 \%$ \\
\hline
\end{tabular}

Tabela 4. Ordem de emissão da artéria escapular dorsal como ramo colateral individual da artéria subclávia, à direita e à esquerda.

\begin{tabular}{ccc}
\hline Ordem de emissão & \multicolumn{2}{c}{ Individualmente } \\
\hline & direita & esquerda \\
$2^{\circ}$ ramo & $3,30 \%$ & $6,70 \%$ \\
$3^{\circ}$ ramo & $10,00 \%$ & $10,00 \%$ \\
$4^{\circ}$ ramo & $16,70 \%$ & $10,00 \%$ \\
$5^{\circ}$ ramo & $6,70 \%$ & $6,70 \%$ \\
\hline
\end{tabular}


Tabela 5. Frequência de emissão da artéria escapular dorsal em tronco comum com outras artérias, como ramo colateral da artéria subclávia, à direita e à esquerda.

\begin{tabular}{|c|c|c|c|c|c|c|c|c|}
\hline \multirow[t]{2}{*}{ Tronco em comum com: } & \multicolumn{2}{|c|}{ como $1^{\circ}$ ramo } & \multicolumn{2}{|c|}{ como $2^{\circ}$ ramo } & \multicolumn{2}{|c|}{ como $3^{\circ}$ ramo } & \multicolumn{2}{|c|}{ como $4^{\circ}$ ramo } \\
\hline & $\mathrm{D}$ & $\mathrm{E}$ & $\mathrm{D}$ & $\mathrm{E}$ & $\mathrm{D}$ & $\mathrm{E}$ & $\mathrm{D}$ & $\mathrm{E}$ \\
\hline Aa. V.T. \& T.I. & $3,30 \%$ & & $3,30 \%$ & & & & & \\
\hline A. Ve. \& Tc.C.S-P; & $3,30 \%$ & & $6,70 \%$ & & & $6,70 \%$ & & \\
\hline Aa V.T., Ve \& Tc. C.S-P & $3,30 \%$ & & & & & & & \\
\hline A. V. T. & & & $10 \%$ & & & & & \\
\hline Aa Ve. \& C.P. & & & $3,30 \%$ & $3,30 \%$ & & & & \\
\hline A. C. P. & & & $3,30 \%$ & & $10 \%$ & & & $3,30 \%$ \\
\hline Tc. C. S-P. & & & & & $13,30 \%$ & & $3,30 \%$ & $43,30 \%$ \\
\hline A. Ve. & & & & $6,70 \%$ & & $3,30 \%$ & & \\
\hline
\end{tabular}

Aa. V.T. \& T.I. = artérias vertebral torácica e torácica interna; A. Ve \& Tc.C.S-P = artéria vertebral e tronco comum lcervical superficial - profunda; Aa. V.T., Ve \& Tc.C.S-P = artérias vertebral torácica, vertebral e tronco comum cervical superficial - profunda; A. V.T. = artéria vertebral torácica; Aa Ve \& C.P. = artérias vertebral e cervical profunda; A. C.P. = artéria cervical profunda; Tc.C.S-P = tronco comum cervical superficial - profunda; A. Ve = artéria vertebral; A.S.D. = artéria subclávia direita; A.S.E. = artéria subclávia esquerda.

vidualmente, a artéria cervical profunda, sendo esta lançada sempre em tronco comum com outras artérias. Em 33,3\% das amostras, tanto à direita como à esquerda, a artéria cervical profunda foi emitida em tronco comum com a artéria cervical superficial, conforme a Tabela 6 .

A artéria cervical superficial, de grande calibre, foi lançada craniolateralmente irrigando a face lateral superficial do pescoço e a musculatura extrínseca do membro torácico da região. À direita, a artéria cervical superficial mostrou-se como terceiro ramo individual da artéria subclávia em $6,7 \%$ e como quarto ramo em $10 \%$ das amostras. Já à esquerda, foi emitida como quarto ramo individual em $20 \%$ das preparações e como quinto ramo em $10 \%$. Nas demais preparações, a artéria cervical superficial foi emitida em tronco comum com outras artérias, tanto à direita como à esquerda, sendo isso já observado nas tabelas anteriores.

\section{DISCUSSÃO}

Em algumas espécies de roedores como: Chinchilla lanigera [1], Cavia porcellus [6,9,10] e Agouti paca [8], o arco aórtico emitiu, como seus ramos colaterais em sequência, um tronco braquicefálico e uma artéria subclávia esquerda, coincidindo com um dos padrões encontrados em nutria. Também em outros roedores como: ratinhos albinos, hamsters sírios e ratões albinos [12] do arco aórtico foi emitido um tronco braquiocefálico, uma artéria carótida comum esquerda e uma artéria subclávia esquerda, sendo isso semelhante com o achado em nutria e na minoria das peças em chinchila [1] e capivara [7].

Para alguns autores, em cutia [3] e capivara [4], do arco aórtico foi lançado apenas um tronco braquiocefálico, não sendo isso observado em nutria ou nos outros roedores pesquisados. Do tronco braquiocefálico foi emitido, em sequência, as artérias carótida comum esquerda, carótida comum direita e subclávia direita na grande maioria das peças em porquinhos-da-índia $[6,9,10]$ e chinchila [1], como também em nutria. Já em ratinhos albinos, hamsters sírios e ratões albinos [12], assim como em poucas peças em chinchila [1], do tronco braquiocefálico foram lançadas apenas as artérias carótida comum direita e subclávia direita, o que também foi visto em nutria em quase metade das amostras.

Com relação a ramificação da artéria subclávia, foi observado em chinchila [1], como também em nutria, uma variação bem significativa quanto a sequência de emissão dos vasos, tanto à direita como à esquerda. Na chinchila, [1] a maior prevalência de emissão foi de medial para lateral, da artéria subclávia direita as artérias: vertebral, escapular dorsal, tronco comum torácica interna - vertebral torácica e tronco 
Tabela 6. Frequência de emissão da artéria cervical profunda em tronco comum com a artéria cervical superficial, como ramo colateral da artéria subclávia, à direita e à esquerda.

\begin{tabular}{|c|c|c|c|c|c|c|c|}
\hline \multicolumn{8}{|c|}{ Tronco comum com a artéria cervical superficial } \\
\hline \multicolumn{2}{|c|}{ como $1^{\circ}$ ramo } & \multicolumn{2}{|c|}{ como $2^{\circ}$ ramo } & \multicolumn{2}{|c|}{ como $3^{\circ}$ ramo } & \multicolumn{2}{|c|}{ como $4^{\circ}$ ramo } \\
\hline $\mathrm{D}$ & $\mathrm{E}$ & $\mathrm{D}$ & $\mathrm{E}$ & $\mathrm{D}$ & $\mathrm{E}$ & $\mathrm{D}$ & $\mathrm{E}$ \\
\hline $3,30 \%$ & & $3,30 \%$ & & & & & \\
\hline $3,30 \%$ & & $6,70 \%$ & & & $6,70 \%$ & & \\
\hline \multicolumn{8}{|l|}{$3,30 \%$} \\
\hline & & $10 \%$ & & & & & \\
\hline & & $3,30 \%$ & $3,30 \%$ & & & & \\
\hline & & $3,30 \%$ & & $10 \%$ & & & $3,30 \%$ \\
\hline & & & & $13,30 \%$ & & $3,30 \%$ & $43,30 \%$ \\
\hline & & & $6,70 \%$ & & $3,30 \%$ & & \\
\hline
\end{tabular}

comum cervical superficial - profunda. No caso da nutria, não houve a formação de tronco comum entre as artérias torácica interna e vertebral torácica, sendo emitidos cinco vasos da artéria subclávia direita. À esquerda, ainda em chinchila [1], a artéria subclávia lançou os mesmos quatro vasos, havendo apenas alteração na sequência de saída destas artérias, sendo o primeiro vaso tronco comum torácica interna - vertebral torácica, seguido das artérias: vertebral, escapular dorsal e tronco comum cervical superficial - profunda. Na nutria, foram observados quatro vasos sendo lançados pela artéria subclávia esquerda, havendo a formação de um tronco comum entre três artérias. Em paca, [8] foram observados como ramos colaterais das artérias subclávias, direita e esquerda, as artérias: vertebral, tronco costocervical, cervical superficial, axilar e torácica interna. Isso foi semelhante ao encontrado em Cavia porcellus $[6,10]$, mas nessa espécie houve a formação de um tronco comum entre as artérias vertebral e escapular dorsal, o que não ocorreu em nutria. Já em ratinhos albinos [12], as artérias subclávias apresentaram como vasos colaterais as artérias: torácica interna, intercostal suprema, cervical profunda e vertebral.

\section{CONCLUSÕES}

O arco aórtico da nutria apresenta dois modelos padrões de ramificação. Em $60 \%$ das peças, o arco aórtico emite como ramos colaterais, em sequência, o tronco braquiocefálico e a artéria subclávia esquerda. E em $40 \%$ das amostras, a sequência de emissão é o tronco braquiocefálico, artéria carótida comum esquerda e artéria subclávia esquerda.

A artéria subclávia direita emite normalmente como ramos colaterais sequenciais, em seu trajeto intratorácico, as artérias: vertebral torácica, vertebral, torácica interna, escapular dorsal e tronco comum cervical superficial - profunda continuando para o membro torácico direito como artéria axilar.

A artéria subclávia esquerda lança, em seu trajeto intratorácico, as artérias: vertebral torácica, torácica interna, vertebral e tronco comum cervical superficial - profunda - escapular dorsal, continuando para o membro torácico esquerdo como artéria axilar.

\section{NOTAS INFORMATIVAS}

${ }^{1}$ Heparin - Cristália Produtos Químicos Farmacêuticos Ltda., Itapira, São Paulo, Brasil.

${ }^{2}$ Thionembutal - Cristália Produtos Químicos Farmacêuticos Ltda., Itapira, São Paulo, Brasil.

${ }^{3}$ Cola 603 - Bertoncini Ltda. São Paulo, SP, Brasil.

${ }^{4}$ Jet - Acrílico auto polimerizável - Artigos Odontológicos Clássicos Ltda., São Paulo, Brasil.

${ }^{5}$ Lupa com lâmpada LTS - aumento de cinco vezes.

${ }^{6}$ Sony - DSC-F717 - cyder-shot 5.0 mega pixels 


\section{REFERÊNCIAS}

1 Araújo A.C.P., Oliveira J.C.D. \& Campos R. 2004. Ramos colaterais do arco aórtico e suas principais ramificações em chinchila (Chinchilla lanigera). Revista Portuguesa de Ciências Veterinárias. 99: 53-58.

2 Baroffio R.A., De Paolii J.C. \& Fiordelisi A.O. 1979. Nuestra Nutria. 2nd edn. Buenos Aires: Editorial Hemisferio Sur S.A., $159 \mathrm{p}$.

3 Carvalho M.A.M., Miglino M.A., Didio L.J.A. \& MeloA.P.F. 1999. Artérias mesentéricas cranial e caudal em cutias (Dasyprocta aguti). Veterinária Noticias. 2: 17-24.

4 Culau P.O.V., Reckziegel S.H., Lindemann T., Araújo A.C.P. \& Balzaretti F. 2007. Colaterais do arco aórtico da capivara (Hydrochoerus hydrochaeris). Acta Scientiae Veterinariae. 35(1): 89-92.

5 International Committee on Veterinary Gross Anatomical Nomenclature. 2005. Nomina anatomica veterinaria. 5 th edn. New York, 198p.

6 Kabak M. \& Haziroglu R.M. 2003. Subgross investigation of vessels originating from arcus aortae in Guinea-pig (Cavia Porcellus). Anatomia Histologia Embryologia. 32: 362-366.

7 Miglino M., Souza W.M. \& Nascimento A.A. 1983. Contribuição ao estudo dos colaterais calibrosos do arco aórtico na capivara (Hydrochoerus hydrochoeris). In: Resumos do VIII Encontro de Pesquisas Veterinárias (Jaboticabal, Brasil). pp.65-66.

8 Oliveira F.G., Machado M.R.F., Miglino M.A. \& Nogeuira T.M. 2001. Gross anatomical study of the aortic ARC branches of the paca (Agouti paca, Linnaeus, 1766). Brazilian Journal of Veterinary Research and Animal Science. 38(3): 103-105.

9 Quesada R., Madriz F.L. \& Fernandes W.A. 1986. Colaterales del arco aortico del cuilo Cavia porcellus (Rodentia). Revista Biologia Tropical. 34 (2): 303-304.

10 Shively M.J. \& Stump J.E. 1974. The systematic arterial pattern of the guinea pig: the head, thorax and thoracic limb. American Journal of Anatomie. 139 (2): 269-284.

11 Silva F. 1994. Mamíferos silvestres do Rio Grande do Sul. Porto Alegre: Fundação Zoobotânica do Rio Grande do Sul, $282 p$.

12 Sinzinger V.H. \& Hohenecker J. 1972. Arcus aortae bei Nagern (Rodentia). Anatomischer Anzeiger. 132: 341-347. 